\title{
Drawing of Two-Dimensional Irregular Meshes
}

\author{
Alok Aggarwal ${ }^{1}$, S. Rao Kosaraju ${ }^{2 \star}$, and Mihai Pop ${ }^{2}$ \\ 1 IBM Research Division; Solutions Research Center; \\ Block 1, Indian Institute of Technology; \\ Hauz Khas, New Delhi 110016 \\ 2 Department of Computer Science; Johns Hopkins University; \\ Baltimore, Maryland 21218
}

\begin{abstract}
We present a method for transforming two-dimensional irregular meshes into square meshes with only a constant blow up in area. We also explore context invariant transformations of irregular meshes into square meshes and provide a lower bound for the transformation of down-staircases.
\end{abstract}

\section{Introduction}

We investigate the drawing of any vertically-convex irregular mesh graph, $G$, within a square mesh, $G^{\prime}$. The drawing must satisfy the following 2 properties:

1. $G^{\prime}$ must be a planar embedding (no crossings)

2. edges in $G$ must map into rectilinear paths.

In other words, the embedding is an orthogonal planar drawing.

An example of such a drawing is shown in figure 1.

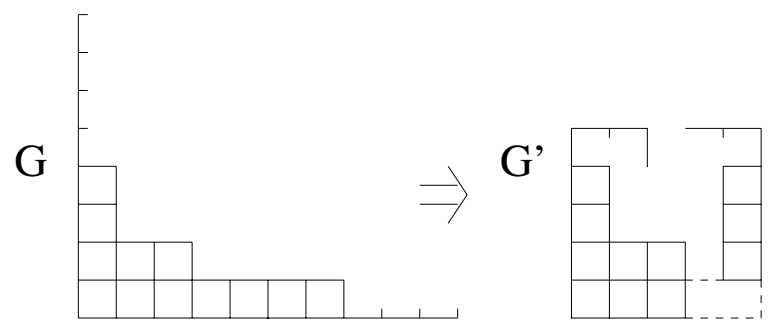

Fig. 1. Mesh transformation

Note that $G^{\prime}$ is planar and every edge of $G$ maps into a path of length $\leq 3$ in $G^{\prime}$.

\footnotetext{
* Supported by NSF Grant CCR9508545 and ARO Grant DAAH04-96-1-0013
} 
The problem of embedding graphs into orthogonal grids has received considerable attention. An annotated list of earlier results can be found in [1. More recently, several authors have dealt with embedding graphs with maximum degree 6 into two dimensional grids, achieving grid areas proportional to the square of the number of vertices [6, 7, 8]. For special cases better results are known. Graphs with maximum degree 6 can be embedded into three dimensional grids of volume $\Theta\left(n^{1.5}\right)$ 3. In the case of trees, two dimensional embeddings of area linear in the number of vertices can be obtained [2, 5, 6].

We address the problem of drawing any vertically-convex mesh (of arbitrary aspect ratio) into a square grid (aspect ratio 1) of area $\Theta(n)$, where $n$ is the number of vertices of $G$. This problem is of great importance in VLSI design. Suppose that a function is realized by interconnecting certain existing VLSI layouts, an example of which is shown in figure 2 If we enclose the figure within the smallest area square (or even a rectangle) then a significant portion of the square might be unused. It is advantageous to transform the figure into a square whose area is proportional to the original wire area and which does not introduce any additional crossings between wires. This problem is solved by our transformation.

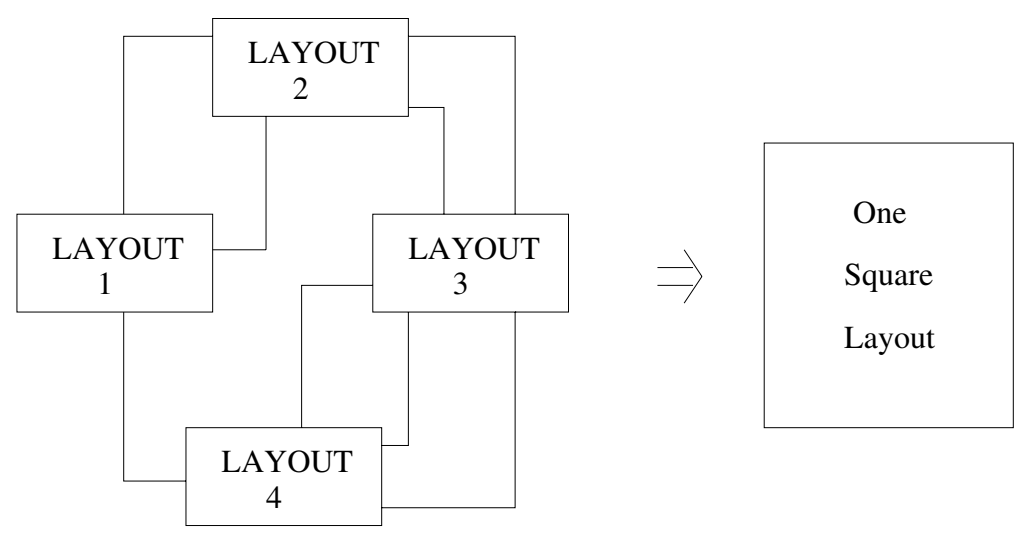

Fig. 2. VLSI layout

Throughout the paper, when we refer to the area $(A)$ of the grid, we mean the footprint area of the mesh, rather than the area of the smallest enclosing rectangle.

In this paper we establish that any vertically-convex mesh of area $A$ can be transformed into an $O(A)$ area square. It was commonly accepted [9] that such a transformation is possible even without imposing the vertical-convexity constraint. However, we could not establish the general result, and our solution to even the specialized class is quite nontrivial.

We then investigate "context-independent transformations" which must satisfy the above two properties, and in addition must satisfy an invariance property 
of Fiat and Shamir [4]. We prove that rectangles of area $\leq A$ can be transformed context-independently into $O(A)$ area squares. We also establish that downstaircases (a very special case of vertically-convex meshes) of area $A$ require $\Omega(A \log A)$ area squares for context-independent transformations. We further prove that any context-independent map that maps down-staircases of area $A$ into $O(A)$ area squares must map $\Omega(\sqrt{\log A})$ nodes of some down-staircase into a single node of the square.

\section{Terminology}

A histogram is a mesh in the first quadrant such that $(0,0)$ is a node, and, if $(x, y)$ is a node, then $(x, y-1),(x, y-2), \ldots,(x, 0) ;(x-1,0),(x-2,0), \ldots,(0,0)$ are also nodes of the histogram. The set of nodes $(0,0),(1,0),(2,0), \ldots$ form the base of the histogram (figure 3). A down-staircase is a histogram in which if $(x, y)$ is a node and $x>0$, then $(x-1, y)$ is also a node of the histogram (figure 4). An up-staircase is defined similarly (figure 51). A monotone histogram is a down- or up-staircase. A double histogram is a mesh in the first and the fourth quadrants in which $(0,0)$ is a node, and if $(x, y)$ is a node then all the grid points between $(x, y)$ and $(x, 0)$ are also nodes of the graph (figure 6). A vertically convex mesh is a mesh in which if $\left(x, y_{1}\right)$ and $\left(x, y_{2}\right)$ are nodes then all the grid points between $\left(x, y_{1}\right)$ and $\left(x, y_{2}\right)$ are also nodes of the graph (figure [7).

\section{Transforming Vertically Convex Meshes}

Lemma 1. There exists a constant $c$ such that any rectangle of area $A$ can be transformed into a square of area $c A[[]$ ].

The proof of the lemma is a simple observation. We can take the rectangle and snake it such that the width of the resulting mesh is roughly $\sqrt{A}$. Folding the rectangle occurs like in figure 8 Finally, we enclose the structure with the smallest possible square. It can be easily shown that the wasted space is at most a fraction of the area of the mesh.

Lemma 2. There exist constants $c_{1}, c_{2}$ and $c_{3}$ such that any down-staircase of area $A$ and base length $l$ can be transformed into a rectangle of area $c_{1} A+c_{2} l^{2}$ and base length $c_{3} l$.

Proof sketch: Enclose the down-staircase within another one such that its "height" for any $x$ in between $2^{i}+1$ and $2^{i+1}$ is the same as the height of the given downstaircase at $2^{i}+1$, as shown in figure 10. The new down-staircase is of area $\leq 2 A$; it is sufficient to consider its transformation. Let the step heights be $d_{0}, d_{1}, \ldots, d_{k}$ as shown. (The area of the original staircase is $A \geq d_{0}+d_{1}+\sum_{i=3}^{k} 2^{i-2} d_{i}$ while the area of the new staircase is $A^{\prime}=d_{0}+d_{1}+\sum_{i=2}^{k} 2^{i-1} d_{i}$. Hence $A^{\prime} \leq 2 A$.) In 
the rest of the proof we consider $d_{i}$ to be the height of the $i^{\text {th }}$ vertical slab and $d_{i}^{\prime}$, as shown in figure 10, to be the height of the $i^{\text {th }}$ horizontal slab.

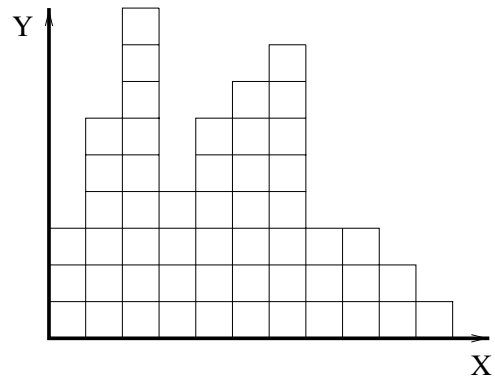

Fig. 3. Histogram

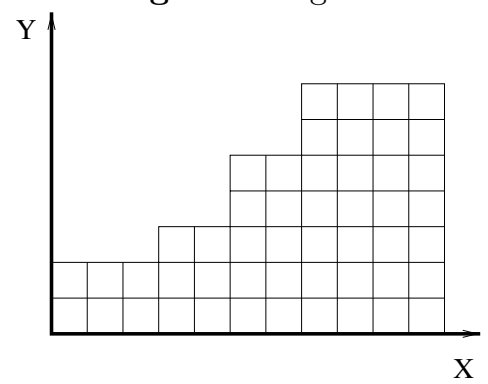

Fig. 5. Up-staircase

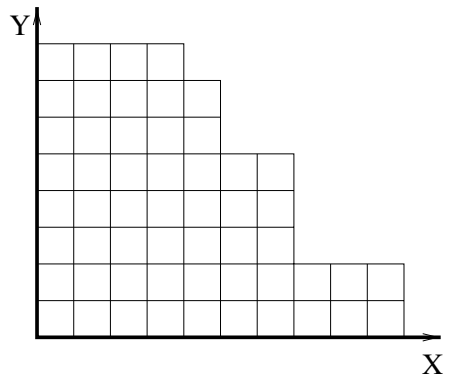

Fig. 4. Down-staircase

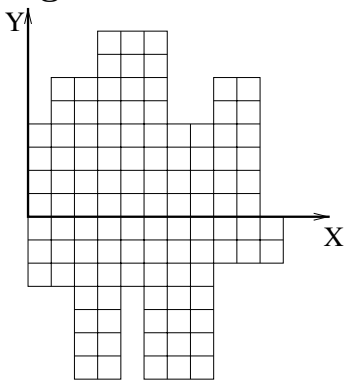

Fig. 6. Double histogram

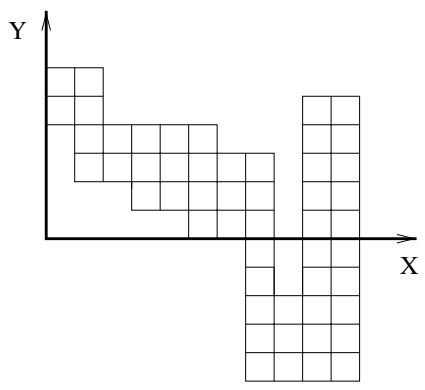

Fig. 7. Vertically-convex mesh

The main idea of the transformation is to fold the new staircase into an almost rectangular shape. For this purpose we consider the staircase decomposed into horizontal slabs as in figure 10. We start by noticing that if all horizontal slabs are "short" we can just enclose the whole staircase in a rectangle of base $l$ and 
height $\sum_{i=0}^{k} d_{i}^{\prime}$. We call a slab "short" if $d_{i}^{\prime} \leq 4 \cdot 2^{i}$. Under the assumption that all slabs are short, the area of the rectangle enclosing the whole staircase is $A_{\text {rectangle }}=2^{k} \sum_{i=1}^{k} d_{i}^{\prime}$. Since $(\forall i), d_{i}^{\prime} \leq 4 \cdot 2^{i}, A_{\text {rectangle }} \leq 4 \cdot 2^{k} \sum_{i=1}^{k} 2^{i} \leq 8 \cdot 2^{2 k}=$ $8 l^{2}$.
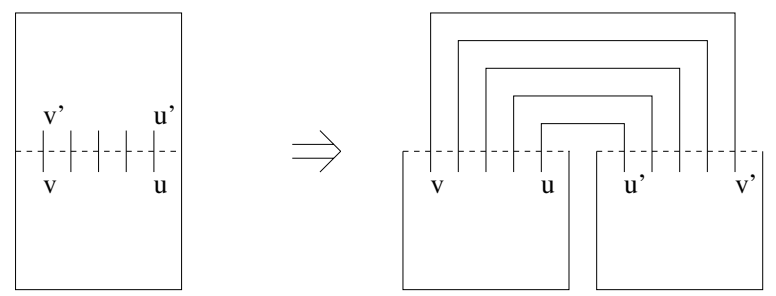

Fig. 8. Folding a rectangle

We now describe a method for transforming a down-staircase into a staircase in which all horizontal slabs are short. The main idea is illustrated in figure 9 . We fold the "tall" slab in such a way that after folding, the slab still properly connects to the two adjacent slabs.

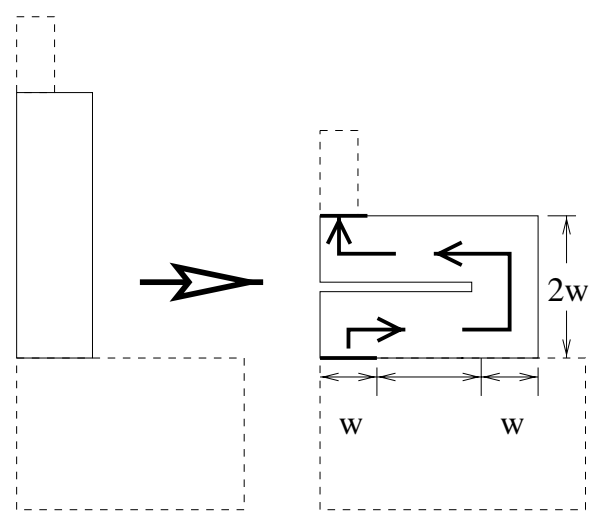

Fig. 9. Folding a "tall" slab

In the following we refer to the height of the current slab as $h$ and the width as $w$. If $h / 2+2 w \leq l$ we only fold once, filling as much as possible of the $l$ width of the rectangle. The rest of the length is wasted space. If $h / 2+2 w>l$ then 
we fold the slab such that it fills the whole $l$ and a portion of height $h-2 l$ is left. We continue this procedure until the leftover part is "short". We have thus transformed a "tall" slab into a short slab and, possibly, a rectangle of width $l$. The folding procedure increases the area of the mesh by only a constant factor due to the definition of a short slab. Therefore, the rectangular portion has an area proportional to that of the original mesh region. We have shown above that the "short" slab regions can be wrapped into a rectangle without blowing up the area by more than $O\left(l^{2}\right)$, thus the total area of the rectangle enclosing the folded staircase is $A^{\prime} \leq c_{1} A+c_{2} l^{2}$.
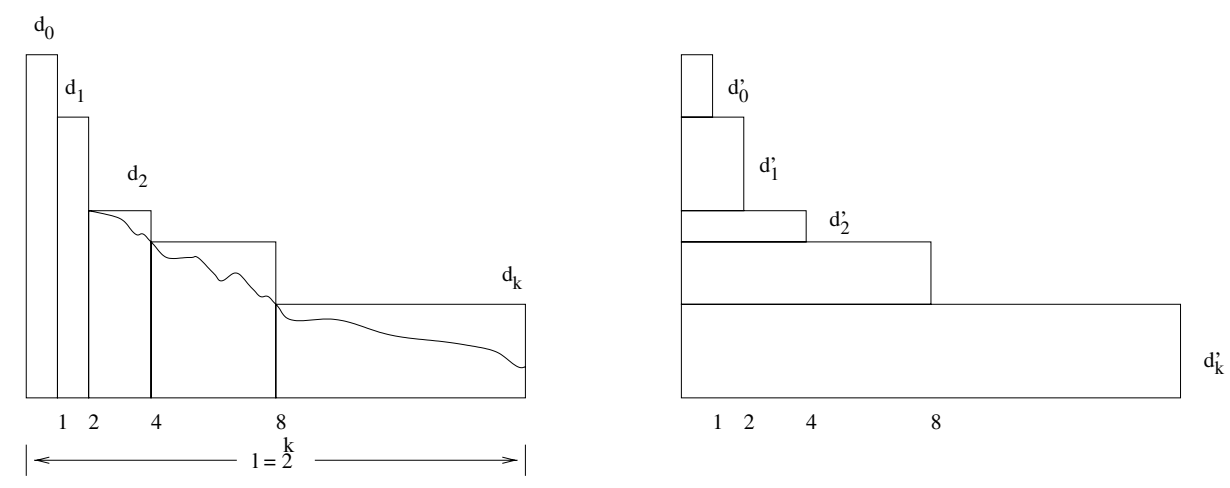

Fig. 10. Staircase

Lemma 3. There exist constants $c_{1}, c_{2}$ and $c_{3}$ such that any histogram of area $A$ and base length $l$ can be transformed into a down-staircase of area $c_{1} A+c_{2} l^{2}$ and of base length $c_{3} l$.

Proof sketch: All the nodes in the $i^{\text {th }}$ row are shifted left by $i / 2$ positions or until they cannot be shifted, as shown schematically in figure 11 A specific example is shown in figure 12. The resulting diagonal lines can be transformed into rectilinear paths as shown in figure 13 This results in $c_{1}=4, c_{2}=4, c_{3}=2$.

As a consequence of Lemmas 2 and 3 we have:

Lemma 4. There exist constants $c_{1}, c_{2}$ and $c_{3}$ such that any histogram of area $A$ and base length $l$ can be transformed into a rectangle of area $c_{1} A+c_{2} l^{2}$ and of base length $c_{3} l$.

We now show how to remove the $l^{2}$ term from the area. Let a histogram consist of $p$ rectangles, the $i^{\text {th }}$ rectangle being $l_{i} \times h_{i}$ as shown in figure 14. 


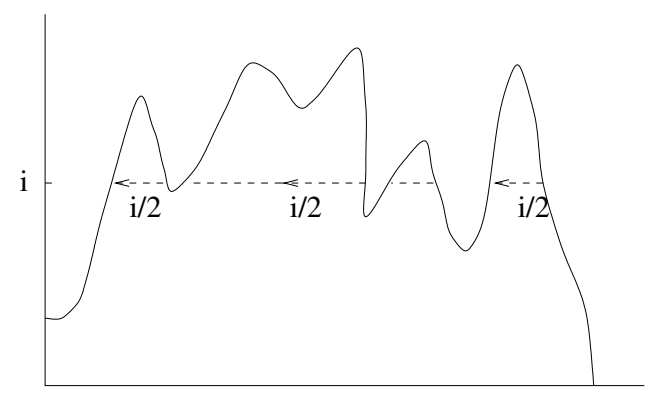

Fig. 11. Conversion of histogram into staircase
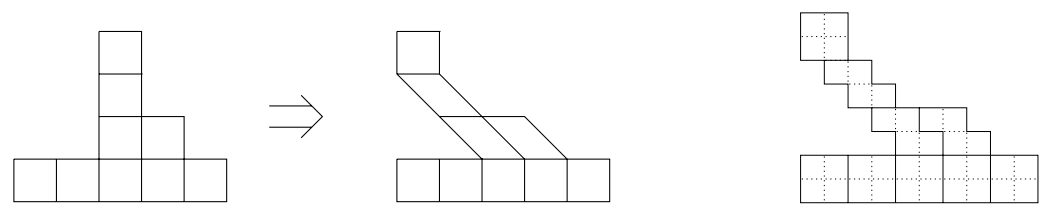

Fig. 12. Example of conversion of his- Fig. 13. Eliminating diagonal lines togram into staircase

Definition: For any $c>0$, the histogram is $c$-skinny if

$$
\sum_{i=1}^{p} h_{i}^{2} \leq c \sum_{i=1}^{p} l_{i} h_{i}
$$

Lemma 5. There exist constants $c_{1}, c_{2}$ and $c_{3}$ such that any histogram of area $A$ and length $l$ can be transformed into a $c_{1}$-skinny histogram of area $c_{2} A$ and length $c_{3} l$.

Proof sketch: Starting from the left, the histogram is partitioned into $p$ sections as follows (see figure 15). For the $i^{\text {th }}$ section, $l_{i}$ is the minimum length such that $A_{i}<l_{i}^{2}$. Then transform the part of $A_{i}$, above the $\max \left\{h_{i-1}, h_{i}\right\}$ row by

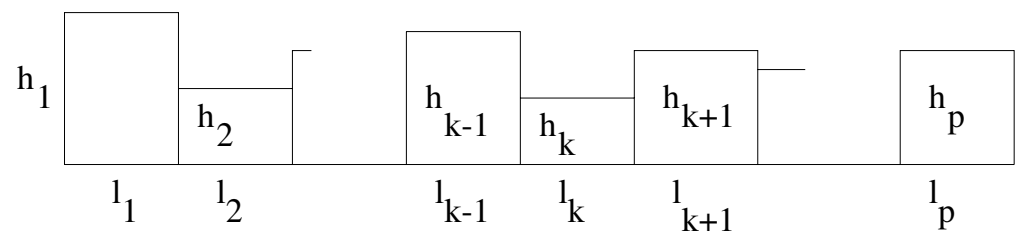

Fig. 14. Histogram 
Lemma 4. Let the new height of the $i^{\text {th }}$ section be $h_{i}^{\prime}$. We can show that

$$
\sum_{i=1}^{p} l_{i} h_{i}^{\prime} \leq c_{2} A \text { and } \sum_{i=1}^{p}{h_{i}^{\prime}}^{2} \leq c_{1} \sum_{i=1}^{p} l_{i} h_{i}^{\prime}
$$

for some constants $c_{1}$ and $c_{2}$.

The first inequality immediately follows from Lemma 4 and the requirement that $A_{i}<l_{i}^{2}$. The second inequality can also be easily proved. We will actually prove a stronger result: $h_{i}^{\prime} \leq c l_{i}$ for some constant $c$. From the transformation of $A_{i}$ we get that $A_{i}^{\prime}=c_{1} A_{i}+c_{2} l_{i}^{2}$ and $l_{i}^{\prime}=c_{3} l_{i}$ (according to Lemma 4). Since $A_{i}^{\prime}$ is the area of a rectangle of height $h_{i}^{\prime}$ and width $l_{i}^{\prime}$, we can write $c_{1} A_{i}+c_{2} l_{i}^{2}=c_{3} l_{i} h_{i}^{\prime}$. But $A_{i}<l_{i}^{2}$ due to the construction and therefore $c_{3} l_{i} h_{i}^{\prime}<c_{1} l_{i}^{2}+c_{2} l_{i}^{2}$. We have, thus, proved that $h_{i}^{\prime}<c l_{i}$ where $c=\left(c_{1}+c_{2}\right) / c_{3}$. The second inequality is now obvious.

First of all we observe that it may be possible that $A>l^{2}$ and there is no position for which $A_{i}<l_{i}^{2}$. In this case we can restrict the histogram to the rows above height $k l$, where $k$ is the largest integer such that $A>k l^{2}$. This may blow up the area of the lower side of the histogram by at most $k l^{2}=\Theta(A)$. We then proceed with the upper side which has area $A^{\prime}<l^{2}$ and are therefore guaranteed to be able to split it as described above.

Second, we restrict the transformation to the section above the $\max \left\{h_{i-1}, h_{i}\right\}$. It is not obvious that the space "wasted" in the lower section is proportional to the area of the pieces, nor that the resulting piece is skinny. The proof of this property is rather technical and is omitted from this preliminary version.

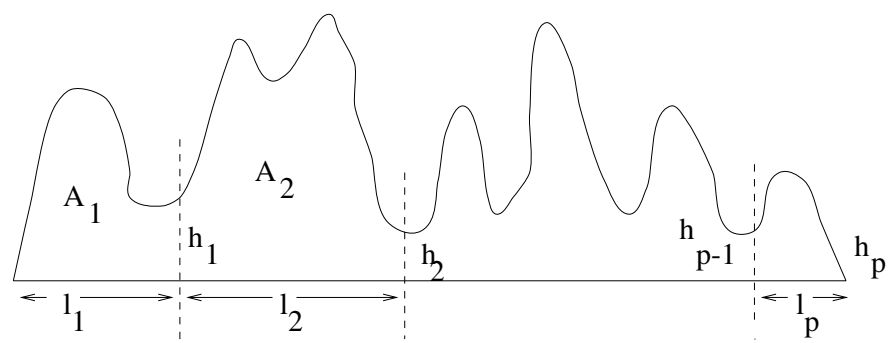

Fig. 15. Partitioning of histogram

Lemma 6. There exists a constant $c_{1}$ such that for any constant $c_{2}$, any $c_{2}$ skinny histogram of area $A$ can be transformed into a rectangle of area $c_{1} c_{2} A$.

Proof sketch: Let the histogram be as shown in figure 14. We can assume without loss of generality that each $h_{k}$ is a power of 3 (simply enclose each region with the smallest rectangle whose height is a power of 3 ; the area can blow up by at most a factor of 3 ). Among $h_{1}, \ldots, h_{p}$ let $h_{k}$ be a smallest height, and let 
$h_{k-1} \leq h_{k+1}$. Split the $l_{k}$ length of the $l_{k} \times h_{k}$ rectangle into $\frac{h_{k-1}}{h_{k}}$ equal length pieces and "fold" them as shown in figure 16

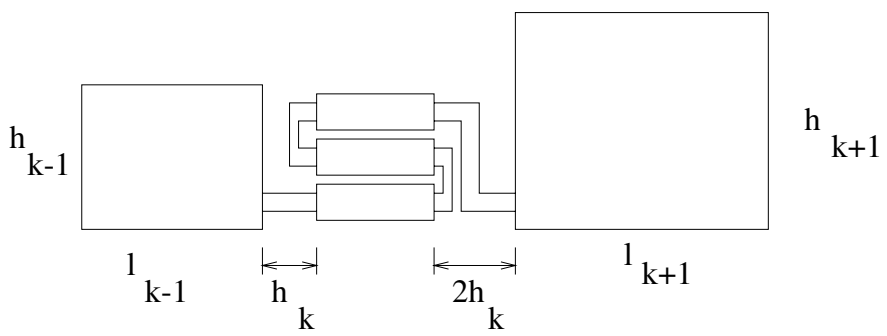

Fig. 16. Folding the histogram into a rectangle

The increase in area can be shown to be $\leq h_{k-1}^{2}$, and it results in the removal of the $h_{k} \times l_{k}$ rectangle. When we iterate this process until only one rectangle remains, we can show that the resulting rectangle satisfies the lemma.

As a consequence of Lemmas [1, 5] and 6] we have

Theorem 1. There exists a constant $c$ such that any histogram of area $A$ can be transformed into a square of area $c A$.

A slightly more complicated construction leads to:

Theorem 2. There exists a constant $c$ such that any double histogram of area $A$ can be transformed into a square of area $c A$.

Lemma 7. There exists a constant $c$ such that any vertically-convex mesh of area $A$ can be transformed into a double histogram of area $c A$.

Proof sketch: Pick any node, $a_{1}$, on the leftmost column. Draw a rectilinear line from $a_{1}$ until the rightmost column is reached such that each horizontal line segment (is left to right and) is as long as possible, and each vertical line segment is as short as possible. Then transform the vertically-convex mesh into a mesh in which the chosen line is horizontal. The idea of the transformation is shown in figure 18. All diagonal lines are embedded onto a grid of suitable size in a manner similar to the approach in figure 13.

As a consequence of Lemma 7 and Theorem 2 we have:

Theorem 3. There exists a constant c such that any vertically convex mesh of area $A$ can be transformed into a square of area $c A$. 


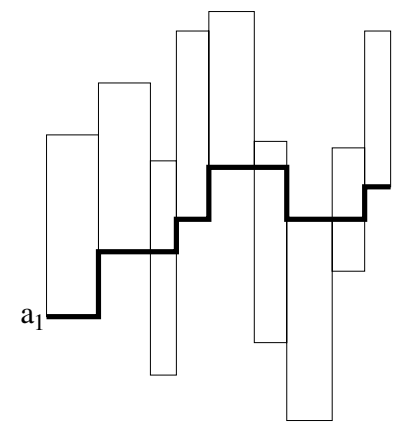

Fig. 17. Transforming a vertically-convex mesh into a double histogram
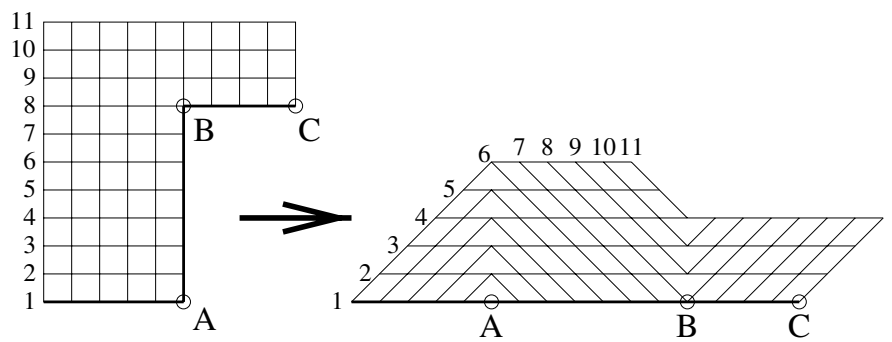

Fig. 18. Idea of transformation

\section{Context-Invariant Transformations}

A planar transformation from a class of graphs into a square is said to be contextinvariant if every 2 nodes, that are in 2 different graphs of the class, that have identical coordinate values w.r.t. the axes, map into the same node of the square.

We saw in Lemma 1 that rectangles of area A can be transformed into $O(A)$ area square. However the transformation is not context-invariant. We show in the next theorem that context-invariant transformations exist for rectangles. Fiat and Shamir 4 gave a nice context-invariant mapping of rectangles of area $\leq A$ into a $O(A)$ area square. (Their final shape is not an exact square but that is not a critical factor). However their mapping results in a non-planar graph.

Consider all the rectangles of area $\leq A$ drawn in the first quadrant such that 2 sides of each rectangle coincide with the 2 axes.

Theorem 4. There exists a constant c such that any rectangle of area $\leq A$ can be transformed context-invariantly into a square of area $c A$.

Every rectangle of area $\leq A$ is a sub-rectangle of one of the $\log A$ rectangles shown in figure 19. Hence it is sufficient to consider the transformation of the $\log A$ rectangles. Observe that any 2 nodes (e.g. $P$ and $Q$ ) which cannot be part of the same rectangle can map into the same node of the square. We fold the 


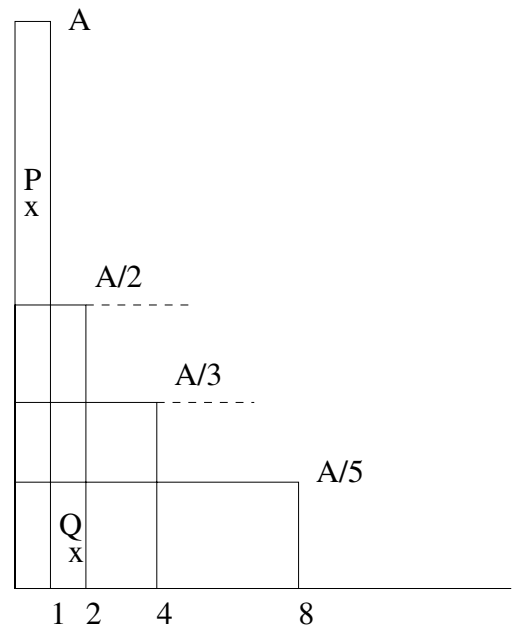

Fig. 19. Staircase containing all rectangles of area $\leq A$

$1 \times A$ rectangle at the $\alpha$-line, and then fold the resulting $2 \times\left(\frac{A}{2}+1\right)$ rectangle at the $\beta$-line, and so on. We stop the folding when the base length is about $\sqrt{A}$. We similarly fold the rectangles of base length $>\sqrt{A}$ toward the $y$-axis. Note that for every two rectangles the overlapping area is folded at the same time, thus insuring the context invariance property.

We can show that these steps form a proper transformation. We might ask whether the result extends to down-staircases.

Theorem 5. Any context-invariant transformation from down-staircases of area $A$ requires an $\Omega(A \log A)$ area square.

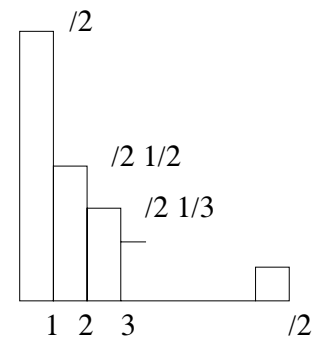

Fig. 20. Transformation from down-staircases of area $\leq A$ requires an $\Omega(A \log A)$ square 
Proof sketch: No two nodes in figure [20] can map into the same node of the square since any two nodes in it can belong to the same down-staircase of area $A$. Hence, the minimum area of the square is $\sum_{i=1}^{A / 2} \frac{A}{2} \frac{1}{i}=\Omega(A \log A)$.

Theorem 6. Any context-invariant mapping from down-staircases to $O(A)$ area squares results in $\Omega(\sqrt{\log A})$ distinct nodes of a down-staircase of area $A$ being mapped into the same node of the square.

Proof sketch: There are $\Omega(A \sqrt{\log A})$ distinct points $(x, y)$ such that $x y \leq \frac{A}{\sqrt{\log A}}$ (since the area of the staircase is $\sum_{i}^{A} \frac{A}{\sqrt{\log A}} \frac{1}{i} \geq \frac{A}{\sqrt{\log A}} \log A=A \sqrt{\log A}$.) Out of these $\Omega(A \sqrt{\log A})$ points, $\Omega(\sqrt{\log A})$ points get mapped into the same point of the square (since the area of the square is $O(A)$.) There exists a down-staircase of area $A$ which includes any $\sqrt{\log A}$ such points.

\section{Achieving Bounded Path Lengths}

Throughout the paper we have not imposed any constraints on the lengths of the paths connecting the original vertices in the embedding. Using the approach from figure 8 the paths can have a length proportional to the width of the mesh.

However the fold can be corrected by keeping the right column edge lengths (like $\left(u, u^{\prime}\right)$ ) at unity and by increasing the lengths of the first $i$ edges of the $i^{\text {th }}$ column (from the right) to 10 as shown in figure 21](due to a minor technicality some of the lengths become 11).

In the following paragraphs we detail the folding procedure. The main idea is to correct the folding scheme of figure 8 . We will fold the vertical paths in the exact way presented in the figure. The horizontal paths, however, will gradually be folded from the $v u$ position to the $v^{\prime} u^{\prime}$ position.

The first step is to quadruple the area of mesh, replacing each original mesh square with four squares the same size as the original. This enables us to connect vertices of the mesh that are not situated on the same row or column. We consider the "mesh"-coordinates of each point in the original mesh to be $(i, j)$, where $i$ is the horizontal coordinate, starting from 0 in the rightmost column, and $j$ is the vertical coordinate, starting from 0 in the row at which we start to fold the mesh. Thus, row 0 is unchanged by the fold, while row 1 is the first row that gets modified.

In the folded mesh, the $j$ coordinate is the distance from the fold along the path on which the original vertical edges were folded (lines $u u^{\prime}, v v^{\prime}$ in figure 8). We denote the new coordinates of $(i, j)$ in the folded mesh by $\left(i^{\prime}, j^{\prime}\right)$. Due to the doubling of the grid, $i^{\prime}=2 i$. In order to achieve a smooth transition in the fold $j^{\prime}$ is described by the following formula:

$$
j^{\prime}= \begin{cases}10 j & j \leq i \\ 8 i+2 j & \text { otherwise }\end{cases}
$$



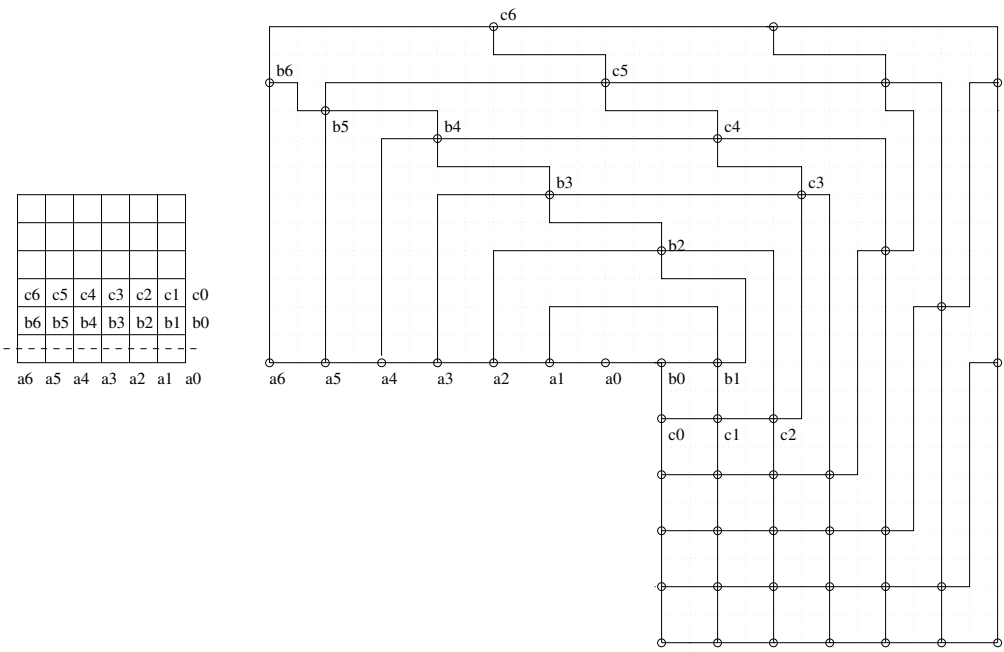

Fig. 21. Actual folding

Whenever $10 j=2 i$ or $10 j=6 i+2$ (vertices $b_{5}$ and $c_{3}$ in figure 21) the vertices would overlap with corners of the folding path. In order to avoid this case we need to subtract 1 from $j^{\prime}$.

It is obvious that the edge lengths along the vertical paths are constant (the mapping function is linear in $j$ ). We only need to show that the horizontal paths are of constant length too. It is easy to see that, for any $j$, and for all $i<j$, the Manhattan distance along the grid between $\left(i^{\prime}, j^{\prime}\right)$ and $\left(i^{\prime}-1, j^{\prime}\right)$ is constant and equal to 2 . This situation occurs in the stable position after the fold. The folded vertical paths "gain" a length of 4 at every $90^{\circ}$ turn. This means that path $i$ needs to be 4 units longer to reach the same position as path $i-1$ (i.e. after the first turn in figure 21, the ends of the paths are vertically aligned). After two $90^{\circ}$ turns the "distance" between adjacent paths increases to 8 , situation captured by the $8 i+2 j$ term in equation 1 Since the original distance between two vertices is equal to 2 , and all vertices are equally spaced on the vertical paths during the fold (the "vertical" distance is 10) the distance between two adjacent vertices $\left(\left(i^{\prime}, j^{\prime}\right)\right.$ and $\left.\left(i^{\prime} \pm 1, j^{\prime}\right)\right)$ is $2+4 \cdot$ number of turns. After two turns the distance is not longer than 10 . The adjustment, made in order to prevent vertices from lying on the corner of a vertical path, only modifies the distance between adjacent vertices by 1 unit (e.g. the distance between $b_{6}$ and $b_{5}$ is 3 instead of 2 and between $b_{5}$ and $c_{5}$ is 11 instead of 10 in figure 21). Therefore, the length of the paths in the folded mesh is still constant.

Similarly we can perform a $90^{\circ}$ fold. The equation for $j^{\prime}$ is:

$$
j^{\prime}= \begin{cases}6 j & j \leq i \\ 4 i+2 j & \text { otherwise }\end{cases}
$$


It follows immediately from the discussion on the $180^{\circ}$ fold that the $90^{\circ}$ fold only increases path lengths by a constant amount.

Using the $90^{\circ}$ and $180^{\circ}$ turn operators we can insure that the path lengths increase only by a constant factor in some of the results presented in the paper. All results, up to lemma 5 inclusively, hold when the two operators are used to insure a constant blow-up in path lengths. For the remaining part of the paper we believe the bound on path lengths holds, however we have not been able to examine all technical details yet. The main difficulty stems from the need to fold the same region several times. The straightforward approach requires a doubling of the mesh dimensions at each fold.

\section{Acknowledgements}

We sincerely thank Professor Mike Goodrich for his many helpful suggestions.

\section{References}

[1] G. Di Battista, P. Eades, R. Tamassia, and I. G. Tollis. Algorithms for drawing graphs: an annotated bibliography, June 1994. electronically available at ftp://wilma.cs.brown.edu/pub/papers/compgeo/gdbiblio.ps.gz.

[2] T. Chan, M. T. Goodrich, S. R. Kosaraju, and R. Tamassia. Optimizing area and aspect ratio in straight-line orthogonal tree drawings. In Proceedings of the Symmposium on Graph Drawing, GD'96, pages 63-75. Springer Verlag, September 1996.

[3] P. Eades, A. Symvonis, and S. Whitesides. Two algorithms for three dimensional orthogonal graph drawing. In Proceedings of the Symmposium on Graph Drawing, GD'96, pages 139-154. Springer Verlag, September 1996.

[4] A. Fiat and A. Shamir. Polymorphic arrays: A novel VLSI layout for systolic computers. In 25th Annual Symposium on Foundations of Computer Science, pages 37-45, Los Angeles, Ca., USA, October 1984. IEEE Computer Society Press.

[5] A. Garg, M. T. Goodrich, and R. Tamassia. Planar upward tree drawings with optimal area. International Journal of Computational Geometry and Applications, 6(3):333-356, 1996.

[6] C. E. Leiserson. Area-efficient graph layouts (for VLSI). In 21st Annual Symposium on Foundations of Computer Science, pages 270-281, Syracuse, New York, 13-15 October 1980. IEEE.

[7] A. Papakostas, J. M. Six, and I. G. Tollis. Experimental and theoretical results in interactive orthogonal graph drawing. In Proceedings of the Symmposium on Graph Drawing, GD'96, pages 371-386. Springer Verlag, September 1996.

[8] A. Papakostas and I. G. Tollis. A pairing technique for area-efficient orthogonal drawings. In Proceedings of the Symmposium on Graph Drawing, GD'96, pages 355-370. Springer Verlag, September 1996.

[9] J. D. Ullman. Computational Aspects of VLSI. Computer Science Press, Maryland, 1984. 